\title{
ARTICLES
}

\section{Negative Fukui functions: New insights based on electronegativity equalization}

\author{
Patrick Bultinck ${ }^{\text {a) }}$ \\ Department of Inorganic and Physical Chemistry, Ghent University, \\ Krijgslaan 281 (S-3), B-9000 Gent, Belgium \\ Ramon Carbó-Dorca \\ Institute of Computational Chemistry, University of Girona, Campus Montilivi, \\ 17071 Girona, Catalonia, Spain \\ Wilfried Langenaeker \\ Johnson \& Johnson Pharmaceutical Research and Development, Molecular Design and Chemoinformatics, \\ Turnhoutseweg 30, B-2340 Beerse, Belgium
}

(Received 7 October 2002; accepted 10 December 2002)

Fukui functions have been calculated for large numbers of organic molecules, and were found to always be positive. Numeric and algebraic considerations allowed the identification of several boundary conditions for negative values for Fukui functions. Negative Fukui functions are found to be very unlikely, except when very short interatomic distances are present. Recent hypotheses concerning the occurrence of negative Fukui functions are strongly supported by the present approach. (C) 2003 American Institute of Physics. [DOI: 10.1063/1.1542875]

\section{INTRODUCTION}

In Fukui's Frontier Molecular Orbital Theory, ${ }^{1,2}$ chemical reactivity toward nucleophiles or electrophiles is interpreted in terms of the HOMO or LUMO electron density. For an electrophilic reaction, the reaction will take place there, where the HOMO electron density is the largest in the molecule. Likewise, for a nucleophilic reaction, the LUMO indicates the preferred site in the molecule.

Within the context of density functional theory (DFT), so-called Fukui functions are introduced, ${ }^{3}$ which are advocated as reactivity descriptors in order to identify the most reactive sites for electrophilic or nucleophilic reactions within a molecule. The most common expression for the Fukui functions is ${ }^{3}$

$$
f(r)=\left(\frac{\partial \rho(r)}{\partial N_{e}}\right)_{V_{\mathrm{ext}}} .
$$

They reflect the change in electron density at a point $r$ with respect to a change in the number of electrons $N_{e}$, under constant external potential $V_{\text {ext }}$. The latter requirement often simply translates in freezing the molecular geometry. Often, one prefers to associate molecular properties like chemical reactivity with atomic entities in the molecule and not with a certain point in space. This means one needs to somehow identify an atom in the molecule. Since there has not yet been defined an operator, which, acting on the wave function or electron density, performs such a division of space in atomic basins, there is no unique definition of an atom in a molecule. Despite this fundamental problem, Fukui functions are often condensed to atomic resolution.

${ }^{a)}$ Electronic mail: Patrick.Bultinck@ rug.ac.be; fax: +32/9/264.49.83.
These condensed Fukui functions are, according to Ayers et al. in the context of a variational approach to chemical reactivity, ${ }^{4-6}$ even more instructive indicators of molecular site reactivity than the actual Fukui function, as introduced in Eq. (1). Atom condensed Fukui functions were first introduced by Yang et al., ${ }^{7}$ using the Mulliken population analysis (MPA). The Fukui function on an atom $\alpha$ is then calculated as the change of the atomic MPA charge with respect to a change in the total number of electrons in the molecule,

$$
f_{\alpha}=-\left(\frac{\partial q_{\alpha}}{\partial N_{e}}\right)_{V_{\text {ext }}} .
$$

These condensed Fukui functions are usually evaluated using a finite difference (FD) methodology considering discrete numbers of electrons. One thus performs calculations for the neutral molecule and for the cationic and anionic species with the same molecular geometry. In FD calculations, three types of Fukui functions are introduced, depending on the species involved, so the following forms are defined:

$$
\begin{aligned}
& f_{\alpha}^{+}=q_{N_{e}}-q_{N_{e}+\Delta N_{e}}, \\
& f_{\alpha}^{-}=q_{N_{e}-\Delta N_{e}}-q_{N_{e}}, \\
& f_{\alpha}^{0}=\frac{1}{2}\left(q_{N_{e}-\Delta N_{e}}-q_{N_{e}+\Delta N_{e}}\right),
\end{aligned}
$$

which correspond to approximations to the actual derivative, from the left and the right, respectively. The Fukui functions $f_{\alpha}^{+}$and $f_{\alpha}^{-}$describe the ability of an atom $\alpha$ to accommodate an extra electron or to cope with the loss of an electron. $f_{\alpha}^{0}$ is then considered as an indicator for radical reactivity. In the above-mentioned calculational scheme, many different errors may be introduced, possibly having an important impact on the resulting values. Among these errors one has in the first 
place the mathematical problem of using the finite difference approximation with $\Delta N_{e}=1$ in Eq. (3). This is quite a crude approximation for the calculation of the derivative. It is claimed that within DFT nonintegral numbers of electrons may be considered. This introduces a number of extra questions of a philosophical nature, like considering obsolete the indivisibility of particles intervening in chemical phenomena. Next to these problems, another crude assumption is that the method used for the neutral molecule is equally well fit for the cationic and anionic species. The DFT functional and basis set should be equally accurate for the neutral molecule, which is often a singlet state, as for charged molecules, which often are doublet states. One further needs to address the way how atoms were defined within the molecule. Usually this directs the problem toward the method used for population analysis.

Several methods have been devised to calculate Fukui functions without using the finite difference approximation. Such approaches include methods based on the calculation of derivatives of the MO coefficients with respect to the number of electrons ${ }^{8}$ and a variational approach proposed by Chattaraj et al. ${ }^{9}$ and implemented by De Proft et al. ${ }^{10}$ using a simple approximation for the hardness kernels. In the present study, the electronegativity equalization method (EEM), as introduced by Mortier et al., ${ }^{11,12}$ is used to calculate atom condensed Fukui functions. In EEM, conceptual DFT quantities, like atom condensed Fukui functions, may be calculated employing separate systems of matrix equations as introduced by Baekelandt et al. ${ }^{13}$ and by Bultinck et al. ${ }^{14}$ An alternative method exists in using algebraic relations between these quantities and the molecular hardness matrix. ${ }^{15}$

A complete discussion of EEM is well outside the scope of the present article, and the reader is referred to the work by Mortier et al. ${ }^{11,12}$ and subsequent work by Bultinck et al. ${ }^{14-18}$ In short, EEM allows the calculation of atomic charges from the hardness matrix. This hardness matrix, within Mortiers EEM, is given for an $\mathrm{N}$-atom molecule by

$$
\mathbf{E}_{\mathbf{0}}=\left[\begin{array}{cccc}
2 \eta_{1}^{*} & r_{12}^{-1} & \cdots & r_{1 N}^{-1} \\
r_{21}^{-1} & 2 \eta_{2}^{*} & \cdots & r_{2 N}^{-1} \\
\vdots & \vdots & \cdots & \vdots \\
r_{N 1}^{-1} & r_{N 2}^{-1} & \cdots & 2 \eta_{N}^{*}
\end{array}\right] .
$$

The symbol $\eta_{i}^{*}$ refers to the so-called effective hardness of atom $i . r_{i j}$ refers to the interatomic distance between atoms $i$ and $j$. The elements of this hardness matrix are, within Mortiers EEM, in fact, the hardness kernels. ${ }^{3}$ It was shown by Bultinck et al. that atomic charges can be calculated from this hardness matrix as ${ }^{15}$

$$
|\mathbf{q}\rangle=\left[\left\langle\mathbf{E}_{0}^{-1}\right\rangle^{-1}\left(\mathbf{E}_{0}^{-1} \mathbf{1}_{0} \mathbf{E}_{0}^{-1}\right)-\mathbf{E}_{0}^{-1}\right]|\chi\rangle+Q\left\langle\mathbf{E}_{0}^{-1}\right\rangle^{-1} \mathbf{E}_{0}^{-1}|\mathbf{1}\rangle .
$$

In this equation, $Q$ denotes the total molecular charge, and $|\chi\rangle$ is a column vector holding the effective electronegativities for all elements. The notation $\left\langle\mathbf{E}_{0}^{-1}\right\rangle$ in Eq. (5), as introduced by Carbó-Dorca et al., ${ }^{19-21}$ refers to the sum of all elements of the hardness matrix inverse. Fukui functions, and many other conceptual DFT quantities can be obtained from EEM. Atom condensed Fukui functions are then given by $^{15}$

$$
|\mathbf{f}\rangle=\left\langle\mathbf{E}_{0}^{-1}\right\rangle^{-1} \mathbf{E}_{0}^{-1}|\mathbf{1}\rangle,
$$

where $|\mathbf{1}\rangle$ is the unity vector, ${ }^{22}$ a column vector containing $N$ elements equal to 1 .

It must be noted here that only one Fukui function is calculated, instead of the three that arise in finite difference approximations. Unfortunately, the effective hardness and electronegativity parameters cannot be obtained directly. They are atoms-in-molecules properties, and were obtained previously from calibration against a large set of molecules representing a wide range of medicinal chemistry. ${ }^{17,18}$ Once these parameters are available, Fukui functions can be calculated directly from Eq. (6) at the very high speed of several millions of molecules/hour on an average PC.

One of the often-cited "problems" with Fukui functions, and their atom condensed versions, is that of negative values. ${ }^{23-26}$ A negative Fukui function value means that when adding an electron to the molecule, in some spots the electron density is reduced, alternatively when removing an electron from the molecule, in some spots the electron density grows larger. This is counterintuitive. Whether such behavior is physically correct is not easily shown, and the only firm requirement consists in that they should sum to one, that is, Fukui functions have to be normalized. ${ }^{3}$ For atom condensed Fukui functions, this last property means that

$$
\langle\mid \mathbf{f}\rangle\rangle=\sum_{\alpha=1}^{N} f_{\alpha}=1 .
$$

Fuentealba et al. have given a number of interesting arguments concerning the non-negativity property, but they also admit the impossibility to truly exclude negative Fukui functions. ${ }^{25,26}$ On the other hand, there are numerical indications that the Hirshfeld charge scheme,${ }^{27}$ based on a stockholder idea, yields only positive Fukui functions. ${ }^{4-6,23,24,28}$ Negative values in other studies are then usually attributed to the characteristics of the population analysis partition scheme used. As was shown previously by Bultinck et al. ${ }^{16}$ for general hardness kernel matrices, even infinite atom condensed Fukui functions are possible when the diagonal dominance of the hardness kernel matrix is lost.

Our previous studies have shown that Fukui functions derived explicitly through EEM via the matrix equations ${ }^{14}$ or using the algebraically derived equations, ${ }^{15}$ are never negative. These tests involved several hundreds of molecules, representing a wide range of medicinal chemistry representative molecular structures. In the present study, the possibility of negative Fukui functions from EEM will be investigated. Starting from the hardness matrix, as given in Eq. (4), numerical tests are performed to observe when Fukui functions are negative, and algebraic derivations will be presented to identify when, for a general polyatomic molecule, negative or even infinite Fukui functions may arise. Also addressed is the question of whether negative Fukui functions are the consequence of using MPA or natural population analysis ${ }^{29}$ (NPA). A recent and interesting study by Ayers et al. ${ }^{6}$ provides the hypothesis stating that diagonal 
dominance of the hardness matrix is sufficient to obtain positive Fukui functions. A general proof of this hypothesis was presented previously by Bultinck et al. ${ }^{16}$ In Mortier's EEM, the hardness kernels $\eta_{\alpha \beta(\neq \alpha)}$ are equal to $r_{\alpha \beta}^{-1}$, and so the diagonal dominance character of the hardness matrix can be manipulated by changing the molecular structure. This allows us to test numerically the importance of diagonal dominance to obtain positive Fukui functions, and to derive new algebraic expressions to identify when Fukui functions are unlikely to be physically meaningful.

\section{RESULTS AND DISCUSSION}

First, several examples and worked-out matrix equations will be presented, whereas in the second section, a set of general, algebraic derivations will be developed, illustrating in a general context when Fukui functions may get negative. As will be shown, negative Fukui functions are not the only counterintuitive events that may occur. Even more counterintuitive is the possibility of infinite Fukui functions that arises as a result of the same theoretical development.

\section{A. Numerical examples and matrix considerations for negative and infinite Fukui functions in small molecules}

In this section several examples will be presented, illustrating the presence of possible discontinuities in the Fukui functions. The first example involves a simple diatomic molecule; the second addresses triatomic molecules. In both cases the EEM matrix equations are worked out explicitly, and those situations where discontinuities are found are examined. In the numerical examples effective hardness parameters are taken from Bultinck et al. ${ }^{17,18}$ These are based on calibrations from MPA. It should, however, be stressed that other population analysis methods, such as $\mathrm{NPA}^{29}$ or Hirshfeld charges, ${ }^{27}$ produce the same conclusions. Furthermore, the algebraic developments do not make any assumption on the method used to obtain these parameters.

\section{Diatomic molecules}

Diatomic molecules are the most tractable case for the study of Fukui function behavior. The hardness matrix in this case, is given by the following formula:

$$
\mathbf{E}=\left[\begin{array}{cc}
2 \eta_{1}^{*} & r_{12}^{-1} \\
r_{21}^{-1} & 2 \eta_{2}^{*}
\end{array}\right]
$$

From previous algebraic results, ${ }^{15}$ it was found that the Fukui function vector is given by

$$
|\mathbf{f}\rangle=\left\langle\mathbf{E}^{-1}\right\rangle^{-1} \mathbf{E}^{-1}|\mathbf{1}\rangle=2 \eta_{M} \mathbf{E}^{-1}|\mathbf{1}\rangle,
$$

where $\eta_{M}$ is the global molecular hardness. It is seen that for the Fukui function evaluation, the hardness inverse matrix is required. For simple molecules it remains tractable to do this in an explicit way:

$$
\mathbf{E}^{-1}=\left[\begin{array}{cc}
2 \eta_{1}^{*} & r_{12}^{-1} \\
r_{21}^{-1} & 2 \eta_{2}^{*}
\end{array}\right]^{-1}=\frac{1}{\Delta}\left[\begin{array}{cc}
2 \eta_{2}^{*} & -r_{12}^{-1} \\
-r_{21}^{-1} & 2 \eta_{1}^{*}
\end{array}\right],
$$

where $\Delta$ denotes the determinant of the hardness matrix. Equation (9) becomes

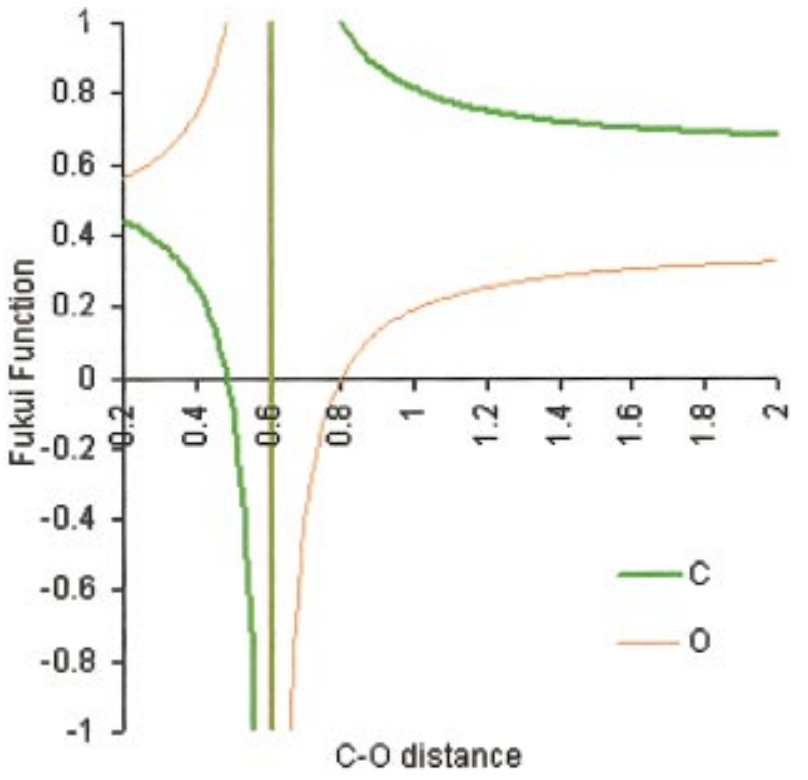

FIG. 1. (Color) Behavior of Fukui functions in $\mathrm{CO}$ as a function of $\mathrm{C}-\mathrm{O}$ interatomic distance (in $\AA$ ).

$$
\begin{aligned}
|\mathbf{f}\rangle & =\left\langle\mathbf{E}^{-1}\right\rangle^{-1} \mathbf{E}^{-1}|\mathbf{1}\rangle \\
& =\left[2\left(\eta_{1}^{*}+\eta_{2}^{*}-r_{12}^{-1}\right)\right]^{-1}\left[\begin{array}{l}
2 \eta_{2}^{*}-r_{12}^{-1} \\
2 \eta_{1}^{*}-r_{21}^{-1}
\end{array}\right] .
\end{aligned}
$$

Consider as a special case any homonuclear diatomic molecule, that is, $\eta_{1}^{*}=\eta_{2}^{*}$. In this case the Fukui functions will be independent of the interatomic distance, and the solution will always be $f_{1}=f_{2}=\frac{1}{2}$, as easily predicted from symmetry considerations. A more interesting case is found when studying heteronuclear diatomic molecules. In this case, a discontinuity is expected when

$$
\left(\eta_{1}^{*}+\eta_{2}^{*}-r_{12}^{-1}\right)=0 .
$$

Taking as an example the $\mathrm{CO}$ molecule, and using the calibrated values for the effective hardness for $\mathrm{C}$ and $\mathrm{O},{ }^{17,18}$ it is easily predicted that a discontinuity should arise near $0.60 \AA$. Doing the EEM calculations using the algebraic expressions given previously, this discontinuity is indeed found. Also, the negative Fukui functions for $\mathrm{C}$ and $\mathrm{O}$ are both found near this discontinuity. It is interesting to note that Fig. 1 shows that this discontinuity and the area where negative Fukui functions are found, occurs at quite small interatomic distances. As the further algebraic development will show, these interatomic distance limits can be obtained from the hardness matrix in a general way. For all distance ranges chemically more common, only positive Fukui functions are found. This fact agrees well with the tests performed by Bultinck et al. for a large set of medicinal molecules, where no negative Fukui functions were found employing EEM, ${ }^{14}$ contrary to the many instances where negative Fukui functions have been found when employing finite difference DFT calculations. In none of the molecules in that study, nor in any of the 138 molecules used in the calibrations of EEM parameters, negative Fukui functions have been found. None of these molecules contained any extraordinarily short interatomic distances either. 
It is interesting to note that Fig. 1 is in fine agreement with the hypothesis by Ayers et al. ${ }^{6}$ and the proof by Bultinck et al. ${ }^{16}$ that diagonal dominance in the hardness matrix yields positive Fukui functions. It is easily checked that diagonal dominance in the present EEM scheme, only occurs starting from $\mathrm{C}-\mathrm{O}$ distances of $0.8 \AA$. The fact that there is also the possibility of positive Fukui functions below this limit is a consequence of numerical aspects, but they should be regarded with skepticism. In those regions the hardness matrix has also lost its positive definite nature, and it is not easy predicting the behavior of the Fukui functions, in general, for a polyatomic molecule below the limit of diagonal dominance. It is worth noting that the Fukui function remains defined within EEM for all distances, but that a discontinuity may arise at some point. Fukui functions remain single valued, and normalization is considered explicitly by including the normalization requirement in the previously derived algebraic relations. ${ }^{15}$

\section{Triatomic molecules}

Triatomic molecules are slightly more involved to derive direct formulas, and, for example, applications; only a number of special cases will be considered in detail. The hardness matrix is given by

$$
\mathbf{E}=\left[\begin{array}{ccc}
2 \eta_{1}^{*} & r_{12}^{-1} & r_{13}^{-1} \\
r_{21}^{-1} & 2 \eta_{2}^{*} & r_{23}^{-1} \\
r_{31}^{-1} & r_{32}^{-1} & 2 \eta_{3}^{*}
\end{array}\right] .
$$

Calculating the inverse explicitly is a straightforward but tedious task. The formulas obtained in the general triatomic case are also relatively little instructive, except in a few simplified cases.

The first of such cases is a simple equilateral triangular molecular geometry, as in cyclopropane with hydrogen atoms omitted. This $D_{3 h}$ structure has the special features consisting in that $\eta_{1}^{*}=\eta_{2}^{*}=\eta_{3}^{*}$ and $r_{12}=r_{23}=r_{13}$. Intuitively, all atoms should have an equal value for the Fukui function, and since Fukui functions sum up to one, the Fukui functions should possess values equal to one-third. Working out Eq. (13) for this special case, one indeed finds this result. More, in general, for all molecules under the special conditions $\eta_{1}^{*}=\eta_{2}^{*}=\cdots=\eta_{N}^{*}$ and all interatomic distances equal, one finds that Fukui functions are equal and have values equal to $1 / N$, independent of the interatomic distances.

Now consider $\mathrm{CO}_{2}$ as a typical linear triatomic molecule. This has the special features consisting of $\eta_{1}^{*}=\eta_{3}^{*}$ $\neq \eta_{2}^{*}$ using an atom numbering as $\mathrm{O}_{1}-\mathrm{C}_{2}-\mathrm{O}_{3}$, and that the following distance relationships hold: $r_{12}=r_{23}=\frac{1}{2} r_{13}$. In this case the Fukui functions are found to be given by

$$
\begin{aligned}
|\mathbf{f}\rangle= & \left\langle\mathbf{E}^{-1}\right\rangle^{-1} \mathbf{E}^{-1}|\mathbf{1}\rangle \\
= & \left(8 \eta_{1}^{*} \eta_{2}^{*}+\left(2 \eta_{1}^{*}\right)^{2}-2\left(4 \eta_{1}^{*}+\eta_{2}^{*}\right) r_{12}^{-1}+\frac{7}{42} r_{12}^{-2}\right)^{-1} \\
& \times\left[\begin{array}{c}
4 \eta_{1}^{*} \eta_{2}^{*}-\left(2 \eta_{1}^{*}+\eta_{2}^{*}\right) r_{12}^{-1}+\frac{1}{2} r_{12}^{-2} \\
\left(2 \eta_{1}^{*}\right)^{2}-4 \eta_{1}^{*} r_{12}^{-1}+\frac{3}{4} r_{12}^{-2} \\
4 \eta_{1}^{*} \eta_{2}^{*}-\left(2 \eta_{1}^{*}+\eta_{2}^{*}\right) r_{12}^{-1}+\frac{1}{2} r_{12}^{-2}
\end{array}\right] .
\end{aligned}
$$

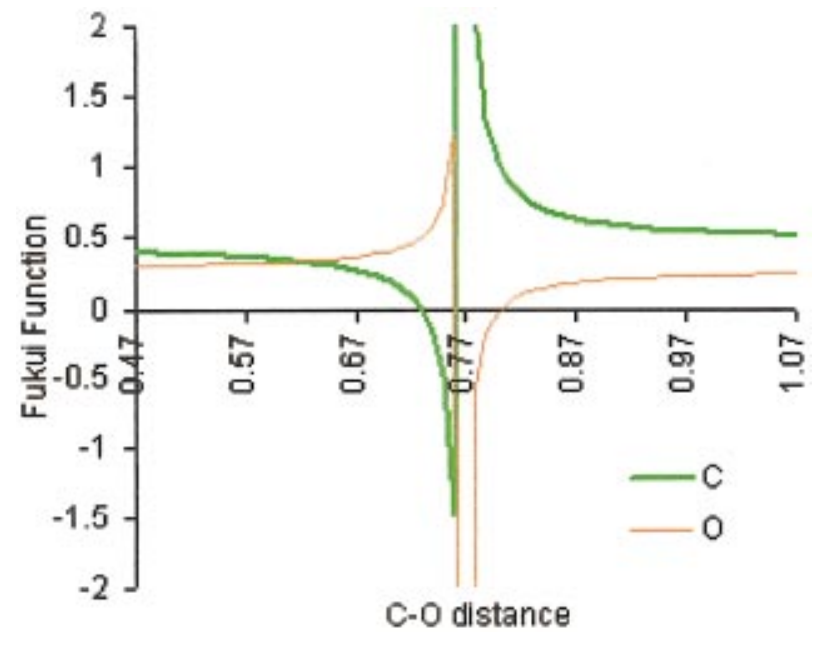

FIG. 2. (Color) Fukui functions in $\mathrm{CO}_{2}$ as a function of $\mathrm{C}-\mathrm{O}$ distance (in $\AA$ ).

Again discontinuities will arise if the denominator in Eq. (14) becomes zero. This yields a quadratic equation in terms of the distance $r_{12}$, and in the specific case of $\mathrm{CO}_{2}$, one of the discontinuities is expected near a $\mathrm{C}-\mathrm{O}$ distance of approximately $0.76 \AA$. This is also found numerically, as shown in Fig. 2.

The negative Fukui functions are found near the discontinuities only, cases where it is also impossible to obtain Fukui functions with good precision. Note that again the discontinuity arises as a consequence of a geometry, where the interatomic distances between carbon and oxygen are significantly smaller than the equilibrium distance, which is approximately $1.17 \AA$. Again, from the moment that diagonal dominance is recovered, the Fukui functions become positive, which agrees with the hypothesis of Ayers et al. ${ }^{6}$ and the proof of Bultinck et al. ${ }^{16}$ The last special case that is easily considered with explicit formulas is found for triatomic molecules where $\eta_{1}^{*}=\eta_{3}^{*} \neq \eta_{2}^{*}$, and $r_{12}=r_{23}$; and the angle $1-2-3$ differs from $180^{\circ}$. A typical example is a $C_{2 V}$ ABA structure like $\mathrm{H}_{2} \mathrm{O}$. Denoting the ABA valence angle as $\alpha$, one has

$$
r_{13}=r_{12} \sqrt{2(1-\cos (\alpha))}=a r_{12} .
$$

The Fukui functions then are given by

$$
\begin{aligned}
|\mathbf{f}\rangle= & \left\langle\mathbf{E}^{-1}\right\rangle^{-1} \mathbf{E}^{-1}|\mathbf{1}\rangle \\
= & \left(8 \eta_{1}^{*} \eta_{2}^{*}+\left(2 \eta_{1}^{*}\right)^{2}-4\left(2 \eta_{1}^{*}+\eta_{2}^{*} a^{-1}\right) r_{12}^{-1}\right. \\
& \left.+\left(4 a^{-1}-a^{-2}\right) r_{12}^{-2}\right)^{-1} \\
& \times\left[\begin{array}{c}
4 \eta_{1}^{*} \eta_{2}^{*}-\left(2 \eta_{1}^{*}+2 \eta_{2}^{*} / a\right) r_{12}^{-1}+a^{-1} r_{12}^{-2} \\
\left(2 \eta_{1}^{*}\right)^{2}-4 \eta_{1}^{*} r_{12}^{-1}+\left(2 a^{-1}-a^{-2}\right) r_{12}^{-2} \\
4 \eta_{1}^{*} \eta_{2}^{*}-\left(2 \eta_{1}^{*}+2 \eta_{2}^{*} / a\right) r_{12}^{-1}+a^{-1} r_{12}^{-2}
\end{array}\right] .
\end{aligned}
$$

Then one can solve this equation for different $\mathrm{O}-\mathrm{H}$ bond lengths and angles $\alpha$. Experimental and calculated data for $\mathrm{H}_{2} \mathrm{O}$ give bond distances around $0.97 \AA$ and the $\mathrm{HOH}$ valence angle around $105^{\circ}$. Investigating the behavior of the Fukui function as a function of the $\mathrm{O}-\mathrm{H}$ distance under the fixed angle $\alpha=105^{\circ}$, a discontinuity is found at very small 


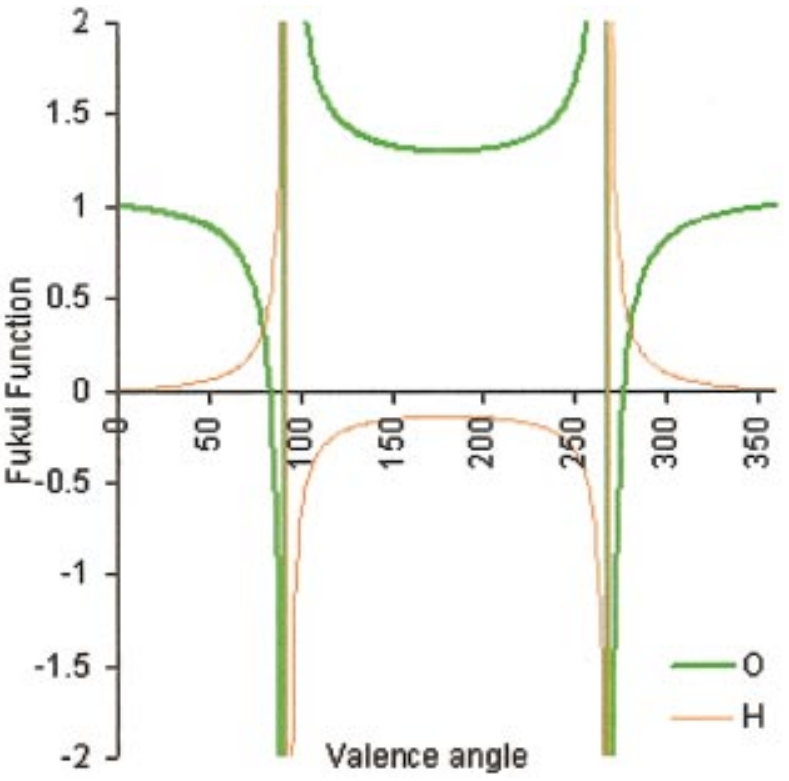

FIG. 3. (Color) Fukui functions as a function of the $\mathrm{H}-\mathrm{O}-\mathrm{H}$ valence angle (in degrees) in $\mathrm{H}_{2} \mathrm{O}$.

$\mathrm{H}-\mathrm{O}$ bond lengths $(0.52 \AA)$. When looking at the $\alpha$ dependence under a fixed $\mathrm{O}-\mathrm{H}$ bond length of $0.97 \AA$, interestingly enough no discontinuities were found, except for the trivial one near $\alpha=0^{\circ}$. Over the entire, chemically reasonable range of valence angles the Fukui functions are always positive, with only minor variation. It is thus found that for geometries that are chemically meaningful, that is, when there are no atoms very close to each other, Fukui functions as obtained through EEM are positive. For $\mathrm{H}_{2} \mathrm{O}$, discontinuities can, however, also appear again in uncommon molecular geometries. As an example, Fig. 3 shows the Fukui functions and molecular hardness as a function of $\alpha$, for a fixed $\mathrm{O}-\mathrm{H}$ distance of $0.5 \AA$. In such heavily distorted geometries, Fukui functions can hardly be obtained accurately, and large regions exist where they are negative. Such geometries are very unlikely to be present in most molecules, since they can probably only be realized at a very high energetic cost. From three-dimensional plots of the Fukui functions against the $\mathrm{O}-\mathrm{H}$ distance and $\mathrm{H}-\mathrm{O}-\mathrm{H}$ valence angle, it was found that all discontinuities and negative Fukui functions are found in areas that unlikely represent structural features that may occur in actual ground state molecular structures.

The examples presented up to now indicate that Fukui functions may show negative values or even infinite values, but that within chemically meaningful geometries, no such situations are likely to occur. They also indicate that whenever the hardness matrix shows diagonal dominance, the Fukui functions are positive. Working out the actual boundaries for a general polyatomic molecule is, however, a very tedious job, so it is well worth seeking a general, algebraic derivation allowing us to identify possible problems with Fukui function discontinuities.

\section{B. General algebraic considerations for negative and infinite Fukui functions}

As the examples above have shown, there are not only instances where Fukui functions may be negative, but also there are discontinuities where Fukui functions may become infinite. In the following section derivations concerning such behavior will be presented. These derivations are somewhat similar to the proof of the diagonal dominance requirement, ${ }^{16}$ but are specifically aimed at Mortier's EEM. ${ }^{11,12}$

The hardness matrix in EEM is defined as follows:

$$
\mathbf{E}=\mathbf{D}+\mathbf{R},
$$

with

$$
\mathbf{D}=\operatorname{Diag}\left\{2 \eta_{I}^{*}\right\} \wedge \mathbf{R}=\left\{\delta(I \neq J) r_{I J}^{-1}\right\},
$$

$\left\{2 \eta_{I}^{*}\right\}$ being the effective hardness elements, $\delta(I \neq J)$ a logical Kronecker delta ${ }^{21,30-34}$ and $r_{I J}^{-1}$ the inverses of the interatomic distances. Let us first consider the case of a $\mathrm{N}$ atom molecule, with hardness matrix $\mathbf{E}_{\mathbf{0}}$, given explicitly by Eq. (4). When adding an extra atom, the hardness matrix may be written as

$$
\mathbf{E}=\left[\begin{array}{cc}
\mathbf{E}_{\mathbf{0}} & |\mathbf{r}\rangle \\
\langle\mathbf{r} & 2 \eta_{N+1}^{*}
\end{array}\right]
$$

$|\mathbf{r}\rangle$ is the column vector holding the elements $r_{i(N+1)}^{-1}(i$ $=1, \ldots, N)$, representing the reciprocals of the distance between the $\mathrm{N}$ atoms on the one hand and the added atom on the other hand. $\eta_{N+1}^{*}$ is the effective hardness of the added atom.

Fukui functions for the $\mathrm{N}$ atom molecule are given algebraically by Eq. (6). For the $N+1$ molecule one then has

$$
|\mathbf{f}\rangle=\left\langle\mathbf{E}^{-1}\right\rangle^{-1} \mathbf{E}^{-1}\left[\begin{array}{c}
|\mathbf{1}\rangle \\
1
\end{array}\right]=2 \eta_{M} \mathbf{E}^{-1}\left[\begin{array}{c}
|\mathbf{1}\rangle \\
1
\end{array}\right],
$$

where $\eta_{M}$ is the global molecular hardness. Thus, the hardness matrix is a matrix with positive definite elements and the molecular hardness is a positive scalar as well. The Fukui function vector has to fulfill the additional property consisting in that the sum of its elements shall be equal to one, that is,

$$
\langle\mid \mathbf{f}\rangle\rangle=\sum_{I=1}^{N+1} f_{I}=1 .
$$

Then, it is not an out of context conjecture to suppose that the Fukui function vector elements, also become positive definite, once the system (20) is solved. Numerical tests over a large set of molecules behave according to this positive definite trend for the elements of the Fukui function vector. ${ }^{14}$ On the other hand, unfortunately, nothing proves that the above conjecture is always fulfilled. There also seems to be no clues about the possibility of knowing when any Fukui function element becomes zero or negative. This is so, because from the direct solution of Eq. (20) there is no indication that can provide a proof for this property, as the solved equation by means of the inverse of the hardness matrix, $\mathbf{E}^{-1}=\left\{E_{I J}^{(-1)}\right\}$, only proves that the Fukui function vector elements can be, in general, expressed as sums of the hardness inverse matrix elements,

$$
f_{I}=2 \eta_{M} \sum_{J=1}^{N+1} E_{I J}^{(-1)},
$$


and, from this algorithm, nothing can tell that the Fukui function values are positive definite in any case, as the inverse hardness elements can be negative and the sum could perhaps add up to a negative as well.

Suppose now that Eq. (6) has been previously solved, and thus $\mathbf{E}_{0}^{-1}$ is known. Also suppose that the Fukui functions of this system are all positive. One can then consider when the discontinuities will arise after adding the extra atom. It is clear that to solve for the Fukui functions in the $N+1$ atom molecule, the inverse of the hardness matrix has to be found in the same way that the previous inverse has to be known to solve Eq. (20). The inverse of the symmetric matrix $\mathbf{E}$ in Eq. (19) may be written by means of

$$
\mathbf{E}^{-1}=\left[\begin{array}{cc}
\mathbf{E}_{\mathbf{0}} & |\mathbf{r}\rangle \\
\langle\mathbf{r} & 2 \eta_{N+1}^{*}
\end{array}\right]^{-1},
$$

which, given the symmetric nature of $\mathbf{E}$, may be written as

$$
\mathbf{E}^{-1}=\left[\begin{array}{cc}
\mathbf{E}_{\mathbf{0}}^{[-1]} & \left|\mathbf{e}^{[-1]}\right\rangle \\
\left\langle\mathbf{e}^{[-1]}\right| & e^{[-1]}
\end{array}\right] .
$$

In order to obtain the inverse, one needs to solve

$$
\left[\begin{array}{cc}
\mathbf{E}_{\mathbf{0}}^{[-1]} & \left|\mathbf{e}^{[-1]}\right\rangle \\
\left\langle\mathbf{e}^{[-1]}\right| & e^{[-1]}
\end{array}\right]\left[\begin{array}{cc}
\mathbf{E}_{\mathbf{0}} & |\mathbf{r}\rangle \\
\langle\mathbf{r}| & 2 \eta_{N+1}
\end{array}\right]=\left[\begin{array}{cc}
\mathbf{I}_{\mathbf{0}} & |\mathbf{0}\rangle \\
\langle\mathbf{0}| & 1
\end{array}\right],
$$

where $|\mathbf{0}\rangle$ and $\langle\mathbf{0}|$ are $N$-dimensional column and row zero vectors. The inverse column $\left|\mathbf{e}^{[-1]}\right\rangle$ and row $\left\langle\mathbf{e}^{[-1]}\right|$ are $N$-dimensional vectors, related by transposition. $e^{[-1]}$ is simply a scalar. Note the important difference between $\mathbf{E}_{\mathbf{0}}^{[-1]}$ and $\mathbf{E}_{\mathbf{0}}^{-1}$, which, respectively, are the $(N \times N)$ matrix block in the partitioned matrix, Eq. (24), and the inverse of the hardness matrix $\mathbf{E}_{\mathbf{0}}$. Working out Eq. (25), the following four equations are obtained:

$$
\begin{aligned}
& \mathbf{E}_{0}^{[-1]} \mathbf{E}_{0}+\left|\mathbf{e}^{[-1]}\right\rangle\langle\mathbf{r}|=\mathbf{I}_{0}, \\
& \mathbf{E}_{0}^{[-1]}|\mathbf{r}\rangle+2 \eta_{N+1}^{*}\left|e^{[-1]}\right\rangle=|\mathbf{0}\rangle, \\
& \left\langle\mathbf{e}^{[-1]}\right| \mathbf{E}_{0}+e^{[-1]}\langle\mathbf{r}|=\langle\mathbf{0}|, \\
& \left\langle\mathbf{e}^{[-1]} \mid \mathbf{r}\right\rangle+e^{[-1]} 2 \eta_{N+1}^{*}=1 .
\end{aligned}
$$

Equation (28) is readily worked out, and introducing

$$
\alpha=\left\langle\mathbf{r}\left|\mathbf{E}_{\mathbf{0}}^{-\mathbf{1}}\right| \mathbf{r}\right\rangle-2 \eta_{N+1}^{*},
$$

one finds by substitution in Eq. (29),

$$
e^{[-1]}=-\alpha^{-1} \text {. }
$$

This result, back-substituted in Eq. (28), gives an expression for $\left\langle\mathbf{e}^{[-1]}\right.$,

$$
\left\langle\mathbf{e}^{[-1]}\right|=\alpha^{-1}\langle\mathbf{r}| \mathbf{E}_{\mathbf{0}}^{-1} .
$$

An application of these results in Eq. (26) provides an algorithm to compute the inverse element $\mathbf{E}_{0}^{[-1]}$,

$$
\mathbf{E}_{\mathbf{0}}^{[-1]}=\mathbf{E}_{\mathbf{0}}^{-\mathbf{1}}-\alpha^{-1}\left(\mathbf{E}_{\mathbf{0}}^{-\mathbf{1}}|\mathbf{r}\rangle\langle\mathbf{r}| \mathbf{E}_{\mathbf{0}}^{-\mathbf{1}}\right) .
$$

Finally, one finds for the inverse matrix $\mathbf{E}^{-1}$,

$$
\mathbf{E}^{-1}=\alpha^{-1}\left[\begin{array}{cc}
\alpha \mathbf{E}_{\mathbf{0}}^{-1}-\mathbf{E}_{\mathbf{0}}^{-1}|\mathbf{r}\rangle\langle\mathbf{r}| \mathbf{E}_{\mathbf{0}}^{-1} & \mathbf{E}_{\mathbf{0}}^{-1} \mid \mathbf{r} \\
\langle\mathbf{r}| \mathbf{E}_{\mathbf{0}}^{-1} & -1
\end{array}\right] .
$$

Using the previously derived algebraic relations for the Fukui functions, ${ }^{15}$ one can also readily write

$$
|\mathbf{f}\rangle=2 \eta_{M} \mathbf{E}^{-1}\left[\begin{array}{c}
|\mathbf{1}\rangle \\
1
\end{array}\right],
$$

where $\eta_{M}$ is the global molecular hardness. Shifting the molecular hardness to the left side of the equation, one can equally consider the softness vector, defined as

$$
|\mathbf{s}\rangle=\mathbf{E}^{-1}\left[\begin{array}{c}
|\mathbf{1}\rangle \\
1
\end{array}\right] .
$$

Let us now investigate when discontinuities will occur in the Fukui functions or in the local softnesses. Using Eq. (34) in Eq. (35), one finds

$$
|\mathbf{f}\rangle=2 \eta_{M} \alpha^{-1}\left[\begin{array}{c}
\left(\alpha \mathbf{E}_{\mathbf{0}}^{-\mathbf{1}}-\mathbf{E}_{\mathbf{0}}^{-\mathbf{1}}|\mathbf{r}\rangle\langle\mathbf{r}| \mathbf{E}_{\mathbf{0}}^{-\mathbf{1}}\right)|\mathbf{1}\rangle+\mathbf{E}_{\mathbf{0}}^{-\mathbf{1}}|\mathbf{r}\rangle \\
\left\langle\mathbf{r}\left|\mathbf{E}_{\mathbf{0}}^{-\mathbf{1}}\right| \mathbf{1}\right\rangle-1
\end{array}\right] .
$$

Discontinuities will now arise when the following condition is met:

$$
\alpha=\left\langle\mathbf{r}\left|\mathbf{E}_{\mathbf{0}}^{-\mathbf{1}}\right| \mathbf{r}\right\rangle-2 \eta_{N+1}^{*}=0,
$$

which identifies the point of the discontinuity as

$$
\left\langle\mathbf{r}\left|\mathbf{E}_{\mathbf{0}}^{-1}\right| \mathbf{r}\right\rangle=2 \eta_{N+1}^{*},
$$

or, working out the matrix products,

$$
\sum_{I=1}^{N} \sum_{J=1}^{N} r_{(N+1) I}^{-1} E_{0 ; I J}^{(-1)} r_{J(N+1)}^{-1}=2 \eta_{N+1}^{*} .
$$

Near this point, Fukui functions are very unlikely to be obtained with good precision, and negative values may arise. If we consider as an example again the case of $\mathrm{CO}$, the behavior as in the worked-out example, given above, is reproduced. Formula (40) is, however, a general equation, allowing also identifying those cases where in any polyatomic molecule, discontinuities and negative Fukui functions are likely to arise.

Further support for Eq. (40) as a criterion for discontinuities in Fukui functions is found by Cholesky decomposition $^{35}$ of the hardness matrices $\mathbf{E}_{\mathbf{0}}$ and $\mathbf{E}$. Suppose that the hardness matrix is positive definite in the usual matrix algebra sense: ${ }^{36}$

$$
\mathbf{E}>0 \rightarrow \forall|\mathbf{x}\rangle \neq \mathbf{0}:\langle\mathbf{x}|\mathbf{E}| \mathbf{x}\rangle>0 .
$$

That the definition (41) holds for the hardness matrix can be easily seen, when one considers the nature of the matrix $\mathbf{R}$ entering definition (17), as constructed in Eq. (18). Indeed, inverse distances behave as cosines, ${ }^{37,38}$ and cosines can be considered just as normalized scalar products. Thus, the hardness matrix can be considered, in full, as representing a positive definite metric, provided that the distance part $\mathbf{R}$ corresponds to reasonable interatomic distance values. Of course, this situation can be distorted, and such a deviation will be discussed next and used to examine the nature of the Fukui function vector.

Admitting, as a suitable work hypothesis, the positive definite nature of the hardness matrix $\mathbf{E}$, then any partition of 
the matrix, like in Eq. (19) provides a positive definite submatrix: $\mathbf{E}_{0}$. Then there exists the following Cholesky triangular decomposition: ${ }^{35}$

$$
\mathbf{E}_{0}=\mathbf{T}_{0}^{T} \mathbf{T}_{0},
$$

$\mathbf{T}_{0}$ being the so-called Cholesky decomposition matrix, which is defined as an upper triangular matrix, whose nonnull elements can be computed in terms of those of the original matrix $\mathbf{E}_{0}$, being $\mathbf{T}_{0}^{T}$ the lower triangular transpose of $\mathbf{T}_{0}$. The hardness submatrix is in this manner nonsingular, and the inverse is readily computed as

$$
\mathbf{E}_{0}^{-1}=\mathbf{T}_{0}^{-1} \mathbf{T}_{0}^{-T} .
$$

Moreover, the inverse of a given triangular matrix is also computed by a simple algorithm. Both processes, the triangular matrix decomposition and inversion, are related by a recursive algorithm too, ${ }^{39}$ which can be applied to the augmented row hardness matrix. That is, one can write

$$
\mathbf{E}=\mathbf{T}^{T} \mathbf{T} \rightarrow \mathbf{T}=\left(\begin{array}{cc}
\mathbf{T}_{0} & |\mathbf{t}\rangle \\
\langle\mathbf{0}| & \tau
\end{array}\right),
$$

and a similar structure can be specified for the inverse process,

$$
\mathbf{E}^{-1}=\mathbf{T}^{-1} \mathbf{T}^{-T} \rightarrow \mathbf{T}^{-1}=\left(\begin{array}{cc}
\mathbf{T}_{\mathbf{0}}^{-1} & \left|\mathbf{t}^{(-1)}\right\rangle \\
\langle\mathbf{0}| & \tau^{-1}
\end{array}\right),
$$

with the easily deducible definitions

$$
\begin{aligned}
& |\mathbf{t}\rangle=\mathbf{T}_{\mathbf{0}}^{-\mathbf{T}}|\mathbf{r}\rangle, \\
& \tau^{2}=2 \eta_{N+1}^{*}-\langle\mathbf{t} \mid \mathbf{t}\rangle=2 \eta_{N+1}^{*}-\left\langle\mathbf{r}\left|\mathbf{E}_{0}^{-1}\right| \mathbf{r}\right\rangle,
\end{aligned}
$$

for the Cholesky decomposition matrix and the following algorithm:

$$
\left|\mathbf{t}^{(-1)}\right\rangle=-\tau^{-1} \mathbf{T}_{0}^{-1}|\mathbf{t}\rangle
$$

giving the inverse element to be computed. In the deduction of the above expressions it is supposed that the Cholesky decomposition and its inverse are already known for the previous recursive step involving the hardness submatrix $\mathbf{E}_{0}$.

After this, and taking into account the initial hardness matrix partition, the following expression for the full hardness inverse can be found:

$$
\mathbf{E}^{-1}=\left(\begin{array}{cc}
\mathbf{E}_{0}^{-1}+\left|\mathbf{t}^{(-1)}\right\rangle\left\langle\mathbf{t}^{(-1)}\right| & \tau^{-1} \mid \mathbf{t}^{(-1)} \\
\tau^{-1}\left\langle\mathbf{t}^{(-1)}\right| & \tau^{-2}
\end{array}\right) ;
$$

so, it is a matter of algebraic manipulation to construct the Fukui vector in terms of the hardness inverse matrix, as expressed above:

$$
\begin{aligned}
& |\mathbf{f}\rangle=2 \eta_{M}\left(\begin{array}{c}
\left(\mathbf{E}_{0}^{-1}+\left|\mathbf{t}^{(-1)}\right\rangle\left\langle\mathbf{t}^{(-1)}\right|\right)|\mathbf{1}\rangle+\tau^{-1}\left|\mathbf{t}^{(-1)}\right\rangle \\
\tau^{-1}\left\langle\mathbf{t}^{(-1)} \mid \mathbf{1}\right\rangle+\tau^{-2}
\end{array}\right), \\
& f_{N+1}=2 \eta_{M} \tau^{-1}\left(\left\langle\mathbf{t}^{(-1)} \mid \mathbf{1}\right\rangle+\tau^{-1}\right)
\end{aligned}
$$

Using Eqs. (46) and (47), the Fukui function for atom $N$ +1 is also given by the equation

$$
\begin{aligned}
f & =2 \eta_{M} \alpha^{-1}\left(\left\langle\mathbf{r}\left|\mathbf{E}_{0}^{-1}\right| \mathbf{1}\right\rangle-1\right) \\
& =2 \eta_{M}\left(\left\langle\mathbf{r}\left|\mathbf{E}_{0}^{-1}\right| \mathbf{1}\right\rangle-1\right)\left(\left\langle\mathbf{r}\left|\mathbf{E}_{0}^{-1}\right| \mathbf{r}\right\rangle-2 \eta_{N+1}^{*}\right)^{-1},
\end{aligned}
$$

which indicates again the same condition for the discontinuity, namely $\left\langle\mathbf{r}\left|\mathbf{E}_{0}^{-1}\right| \mathbf{r}\right\rangle-2 \eta_{N+1}^{*}=0$. For not too heavily distorted geometries, one may conclude from the present test cases and present and previous ${ }^{16}$ algebraic findings that as long as the molecular geometries are not too heavily distorted, only positive Fukui functions are found. This again can be connected with the recent results by Ayers et al. ${ }^{6}$ These authors found, independently of the present study, that a requirement for obtaining positive Fukui functions consists in that the hardness matrix should be diagonally dominant. Now consider Eq. (4) or (19), in the present EEM context such positive dominance is always ensured as long as no interatomic distances grow too small. Also, when diagonal dominance is present, then such a fact only emphasizes that the eigenvalues of the hardness matrix will become positive definite, and this is only a proof in turn of the matrix becoming positive definite too, and in this way avoiding discontinuities and, so, negative values are finally avoided as well. These findings are also supported by a more general proof of the necessity for diagonal dominance of the hardness matrix. $^{16}$

Ayers $e t$ al. also point out the role of using the Hirshfeld population analysis, based on a stockholder division of the electron density. The fact that, when using the Hirshfeld population analysis, no negative Fukui functions are found, has been confirmed on several occasions. ${ }^{23,24,28}$ It is worth noting that, despite the negative results reported several times concerning the use of Mulliken or NPA charges for the calculation of finite difference Fukui functions, when using the present EEM scheme one always finds positive Fukui functions for not too heavily distorted geometries. This was found to be independent of the kind of charges used for the calibration of the effective electronegativity and hardness parameters. In using the finite difference approach, many sources of problems may arise, such as relaxation or the accuracy of the functional and basis set used for singlet and doublet spin multiplicities, as it was commented on before. More research is obviously needed to examine whether it is justified to state that one of the sources of negative Fukui functions obtained by finite difference approaches is really the nature of the population analysis used. An exception is MPA, for which it has been described that, employing analytical considerations, nothing can be predicted about the sign of the MPA Fukui functions. ${ }^{40}$

\section{CONCLUSION}

The occurrence of negative values for Fukui functions was studied through the electronegativity equalization method. Using algebraic relations between Fukui functions and different other conceptual DFT quantities on the one hand and the hardness matrix on the other hand, expressions were obtained for Fukui functions for several archetypical small molecules. It was found that not only negative Fukui functions are possible, but even that discontinuities may arise. However, both counterintuitive cases are found only in quite uncommon molecular structures, where atoms tend to come very close to each other. Based on EEM calculations for large molecular sets, no negative Fukui functions were 
found, allowing us to hypothesize that EEM-based Fukui functions are positive, except when very distorted geometries occur.

Algebraic derivations based on matrix inversion and Cholesky decomposition permit us to deduce general relations to identify cases where Fukui functions may show discontinuities. A simple formula has been found, permitting us to express when the addition of an extra atom results in discontinuities. The present derivations arrive to the same conclusion as recent studies, namely that diagonal dominance of the hardness matrix results in positive Fukui functions. In the present EEM case, this means that no atoms should approach each other too closely.

\section{ACKNOWLEDGMENTS}

One of the authors (P.B.) wishes to thank Ghent University and the Fund for Scientific Research-Flanders (Belgium) for their grants to the Computational Chemistry group at Ghent University, and acknowledges the European Community-Access to Research Infrastructure Action of the Improving Human Potential Program, allowing the use of the CEPBA infrastructure at the PolyTechnical University of Catalonia (Spain) and the visitors fellowship with the Institute of Computational Chemistry at the University of Girona (Catalonia, Spain). R.C.-D. acknowledges the Foundation M. F. de Roviralta as well as CICYT Project No. SAF2000-223, which have supported this work.

${ }^{1}$ K. Fukui, T. Yonezawa, and H. Shingu, J. Chem. Phys. 20, 722 (1952).

${ }^{2}$ K. Fukui, T. Yonezawa, C. Nagata, and H. Shingu, J. Chem. Phys. 22, 1433 (1954).

${ }^{3}$ R. G. Parr and W. Yang, Density Functional Theory of Atoms and Molecules (Oxford University Press, New York, 1989).

${ }^{4}$ P. W. Ayers and R. G. Parr, J. Am. Chem. Soc. 122, 2010 (2000).

${ }^{5}$ P. W. Ayers and R. G. Parr, J. Am. Chem. Soc. 123, 2007 (2001).

${ }^{6}$ P. W. Ayers, R. C. Morrison, and R. K. Roy, J. Chem. Phys. 116, 8731 (2002).

${ }^{7}$ W. Yang and W. Mortier, J. Am. Chem. Soc. 108, 5708 (1986).

${ }^{8}$ R. Balawender and L. Komorowski, J. Chem. Phys. 109, 5203 (1998).

${ }^{9}$ P. K. Chattaraj, A. Cedillo, and R. G. Parr, J. Chem. Phys. 103, 7645 (1995).

${ }^{10}$ F. De Proft, P. Geerlings, S. Liu, and R. G. Parr, Pol. J. Chem. 72, 1737 (1998).

${ }^{11}$ W. J. Mortier, S. K. Ghosh, and S. Shankar, J. Am. Chem. Soc. 108, 4315 (1986).
${ }^{12}$ W. J. Mortier, Struct. Bonding (Berlin) 66, 125 (1987).

${ }^{13}$ B. G. Baekelandt, G. O. A. Janssens, H. Toufar, W. J. Mortier, and R. A. Schoonheydt, in Acidity and Basicity in Solids: Theory, Assessment and Utility, NATO ASI Series C444, edited by J. Fraissard and L. Petrakis (Kluwer Academic, Dordrecht, 1994), pp. 95-126.

${ }^{14}$ P. Bultinck, W. Langenaeker, R. Carbó-Dorca, and J. Tollenaere, J. Chem. Inf. Comput. Sci. (to be published).

${ }^{15}$ P. Bultinck and R. Carbó-Dorca, Chem. Phys. Lett. 364, 357 (2002).

${ }^{16}$ P. Bultinck and R. Carbó-Dorca, J. Phys. Chem. A (to be published).

${ }^{17}$ P. Bultinck, W. Langenaeker, P. Lahorte, F. De Proft, P. Geerlings, C. Van Alsenoy, and J. Tollenaere, J. Phys. Chem. A 106, 7887 (2002).

${ }^{18}$ P. Bultinck, W. Langenaeker, P. Lahorte, F. De Proft, P. Geerlings, M. Waroquier, and J. Tollenaere, J. Phys. Chem. A 106, 7895 (2002).

${ }^{19}$ R. Carbó and E. Besalú, J. Math. Chem. 18, 37 (1995).

${ }^{20}$ R. Carbó and E. Besalú, Comput. Chem. (Oxford) 18, 117 (1994).

${ }^{21}$ R. Carbó and E. Besalu, Strategies and Applications in Quantum Chemistry, edited by Y. Ellinger and M. Defranceschi (Kluwer Academic, Dordrecht, 1996), pp. 229-248.

${ }^{22}$ R. Carbó-Dorca, THEOCHEM 537, 41 (2001).

${ }^{23}$ R. K. Roy, S. Pal, and K. Hirao, J. Chem. Phys. 110, 8236 (1999).

${ }^{24}$ R. K. Roy, K. Hirao, and S. Pal, J. Chem. Phys. 113, 1372 (2000).

${ }^{25}$ P. Fuentealba, P. Pérez, and R. Contreras, J. Chem. Phys. 113, 2544 (2000).

${ }^{26} \mathrm{P}$. Fuentealba and R. Contreras, "Fukui function in chemistry" (private communication, 2002).

${ }^{27}$ F. L. Hirshfeld, Theor. Chim. Acta 44, 129 (1977).

${ }^{28}$ J. Olah, C. Van Alsenoy, and A. B. Sannigrahi, J. Phys. Chem. A 106, 3885 (2002).

${ }^{29}$ A. E. Reed, R. B. Weinstock, and F. Weinhold, J. Chem. Phys. 83, 735 (1985).

${ }^{30} \mathrm{~A}$ logical Kronecker delta is a symbol with the form $\delta([$ logical expression]), which when the [logical expression] argument becomes "true," then the symbol is considered to return a unit value, on the contrary, the logical Kronecker delta provides a zero if the argument is "false."

${ }^{31}$ R. Carbó and J. M. Riera, Lecture Notes in Chemistry (Springer-Verlag, Berlin, 1978), Vol. 5.

${ }^{32}$ R. Carbó and E. Besalu, J. Math. Chem. 13, 331 (1993).

${ }^{33}$ R. Carbó and E. Besalu, Comput. Chem. (Oxford) 18, 17 (1994).

${ }^{34}$ R. Carbó and E. Besalu, J. Math. Chem. 18, 37 (1995).

${ }^{35}$ R. S. Martin, G. Peters, and J. H. Wilkinson, in Linear Algebra, Handbook for Automatic Computation, edited by J. H. Wilkinson and C. Reinsch (Springer-Verlag, Berlin, 1971), Vol. II, pp. 9-30.

${ }^{36}$ R. Carbó and Ll. Domingo, Algebra Matricial y Lineal (SchaumMcGraw-Hill, Madrid, 1987).

${ }^{37}$ R. Carbó-Dorca, E. Besalú, Ll. Amat, and X. Fradera, in Advances in Molecular Similarity, edited by R. Carbó-Dorca and P. G. Mezey (JAI Press, London, 1996), Vol. 1, pp. 1-42.

${ }^{38}$ R. Carbó, E. Besalú, Ll. Amat, and X. Fradera, J. Math. Chem. 16, 47 (1996).

${ }^{39}$ R. Carbó and E. Besalú, Adv. Quantum Chem. 24, 117 (1992).

${ }^{40}$ R. K. Roy, K. Hirao, S. Krishnamurty, and S. Pal, J. Chem. Phys. 115, 2901 (2001) 